\title{
Adverse Effects of Interferon on the Cardiovascular System in Patients with Chronic Hepatitis $G$
}

\author{
Hiroki TeRagawa,* MD, Tatsuya Hondo, MD, \\ Hajime Amano, MD, Fumiaki Hino, MD, \\ and Makoto Ohbayashi, MD
}

\begin{abstract}
SUMMARY
The therapeutic effects of interferon in chronic hepatitis $C$ and many of its adverse effects have been well documented. However, there are only a few reports regarding its adverse effects on the cardiovascular system. The aim of this study was to clarify the clinical features of the adverse effects of interferon on the cardiovascular system in patients with chronic hepatitis $\mathrm{C}$. We monitored 295 patients with chronic active hepatitis $\mathrm{C}$ during 312 courses of interferon therapy and for 1 year after the end of treatment for the presence of cardiovascular adverse effects. We found 6 patients with cardiovascular adverse effects during interferon therapy and 4 more patients within 1 year after the end of therapy $(10 / 312,3.2 \%)$. The adverse effects of interferon on the cardiovascular system included arrhythmia $(n=4)$, ischemic heart disease $(n=4)$ and myocardial disease $(n=2)$. None of the clinical factors, including history of cardiovascular disease, were related to these cardiovascular adverse effects. In all instances the patient's condition improved after discontinuation of interferon and adequate therapy. The cardiovascular adverse effects of interferon occurred frequently in patients with chronic hepatitis $\mathrm{C}$, even after the end of therapy and they were unpredictable. Thus, all patients undergoing interferon therapy should be monitored not only during but also after the end of treatment. (Jpn Heart J 1996; 37: 905-915)
\end{abstract}

Key words : Chronic active hepatitis C Cardiovascular adverse effects Arrhythmia Ischemic heart disease Myocardial disease

TNERFERON (IFN) is an anti-viral agent that has proved effective as treatment for chronic hepatitis $\mathrm{C}$. ${ }^{1,2)}$ However, many adverse effects have also been reported as the number of patients administered IFN therapy and the total dose of IFN have increased and the duration of therapy has been prolonged. ${ }^{3-6)}$ The most prominent among the adverse effects reported is an influ-

From the Department of Internal Medicine, Onomichi General Hospital, Onomichi, Japan. *Present Institute: First Department of Internal Medicine, Hiroshima University School of Medicine, Hiroshima, Japan.

Address for correspondence: Hiroki Teragawa, MD, First Department of Internal Medicine, Hiroshima University School of Medicine, 1-2-3 Kasumi, Minami-ku, Hiroshima 734, Japan.

Received for publication April 10, 1996.

Accepted July 3, 1996. 
enza-like syndrome characterized by fever and chills. Malaise, fatigue, bone marrow suppression, gastro-intestinal discomfort and neuropsychiatric disorders have been also observed.

As for its cardiovascular adverse effects, only a few cases, among patients with chronic hepatitis $\mathrm{C}$, have been documented. ${ }^{7-11\rangle}$ On the other hand, various cases of cardiovascular adverse effects including arrhythmia, dilated cardiomyopathy and ischemic heart disease have been reported among patients administered IFN for various malignancies. ${ }^{12-22)}$ The purpose of this study was to elucidate the clinical features of the adverse effects of IFN on the cardiovascular system in patients with chronic hepatitis C. We herein report 10 patients who presented with cardiovascular adverse effects not only during but also after the end of IFN therapy.

\section{Methods}

Patients: From February 1992 to July 1994, 295 patients (182 men, 113 women) with chronic active hepatitis $\mathrm{C}$ were administered 312 courses of IFN therapy. The average age of the patients was 51 years (range, 19 to 78 years). Seventeen patients underwent repeated IFN therapy.

Protocol: The types of IFN used in our hospital were natural alpha IFN in 203 instances, recombinant alpha-2b IFN in 66, recombinant alpha-2a IFN in 34 and beta IFN in 9 instances. For alpha IFN, except for alpha-2a IFN, 6 million units were injected daily for the first 2 weeks (203 instances) or longer (max: 8 weeks; 66 instances) and 3 times per week thereafter (up to 24 weeks). For alpha-2a IFN, 9 million units were injected daily for the first 2 weeks and 6 million units 3 times per week for the next 22 weeks. Alpha IFNs were administered intramuscularly, while beta IFN was administrated intravenously at a dose of 6 million units per day, every day, for 6 or 8 weeks. Two hundred and seventy patients completed the whole course but the others did not for various reasons such as complications and ineffectiveness. Response was defined as the normalization of transaminase levels during IFN therapy and maintenance of normal transaminase levels after IFN therapy. A response to IFN therapy was observed in 87 instances (27.9\%).

Cardiovascular adverse effects: Electrocardiograms (ECG) and chest X-ray films were taken routinely before IFN therapy. During and after the start of IFN therapy, various tests such as ECG, echocardiogram, treadmill exercise test and ambulatory electrocardiographic Holter recording were performed when a patient complained of chest symptoms. Cardiovascular adverse effect was defined as a new finding recognized by these tests in patients who had complained of no chest symptoms before IFN therapy. In addition, the possible relationship between some clinical factors and cardiovascular adverse effects was examined. 
Other complications observed during and after IFN therapy: Sixty-four other events, which seemed to be complications of IFN therapy, were seen in 60 patients; they included alopecia $(n=23)$, bone marrow suppression $(n=14)$, depressive psychosis $(n=13)$, general fatigue $(n=3)$, pneumonitis $(n=2)$, tremor $(n=1)$, skin eruption $(n=1)$, hematuria $(n=1)$, dementia $(n=1)$, vertigo $(n=1)$, worsening of diabetes mellitus $(n=1)$, pain due to cholelithiasis $(n=1)$, ophthalmic bleeding $(n=1)$ and herpes zoster $(n=1)$. Of these, IFN therapy was discontinued in 33 instances.

Statistical analysis: Chi-square test and Mann-Whitney's $U$ test were used for the statistical analysis of data. All probability values below 0.05 were considered to be statistically significant.

\section{Results}

Cardiovascular adverse effects were recognized in 6 patients during treatment and in 4 within 1 year after the end of IFN therapy. Thus, cardiovascular adverse effects were seen in 10 cases $(3.2 \%)$ among 312 courses of IFN therapy. These were arrhythmia $(n=4)$, ischemic heart disease $(n=4)$ and myocardial disease $(n=2)$. Among those who had arrhythmias, there were 2 patients with atrial fibrillation (Af), one with sinus bradycardia and one with atrioventricular block. As for ischemic heart disease, 3 patients had vasospastic angina pectoris in which coronary angiography $(\mathrm{CAG})$ revealed no significant stenosis; the other patient had microvascular angina, which was diagnosed based on positive findings in the treadmill exercise test, no significant stenosis on the CAG and im-

Table I. Cardiovascular Adverse Effects of IFN Therapy

\begin{tabular}{|c|c|c|c|c|c|c|}
\hline $\begin{array}{l}\text { Case } \\
\text { No. }\end{array}$ & $\begin{array}{l}\text { Sex/ } \\
\text { Age }\end{array}$ & $\begin{array}{l}\text { Cardiovascular } \\
\text { adverse effect }\end{array}$ & $\begin{array}{c}\text { History of } \\
\text { cardiovascular } \\
\text { cisease }\end{array}$ & $\begin{array}{l}\text { Type of } \\
\text { IFN }\end{array}$ & $\begin{array}{l}\text { Total IFN } \\
\text { dose (MU) }\end{array}$ & Onset \\
\hline \multicolumn{7}{|c|}{ Arrhythmia } \\
\hline 1 & $F / 53$ & Paroxysmal Af & None & Recombinant alpha-2A & 126 & 14 days* \\
\hline 2 & $F / 64$ & Sinus bradycardia & Hypertension & Recombinant alpha- $2 \mathrm{~A}$ & 99 & 11 days* \\
\hline 3 & $F / 62$ & Complete $\mathrm{A}-\mathrm{V}$ block & Hypertension & Recombinant alpha-2A & 522 & 4 months** \\
\hline 4 & $M / 50$ & Paroxysmal Af & None & Recombinant alpha-2B & 186 & 31 days* \\
\hline \multicolumn{7}{|c|}{ Ischemic heart disease } \\
\hline 5 & $\mathrm{M} / 62$ & Vasospastic angina pectoris & None & Recombinant alpha-2A & 522 & 7 months** \\
\hline 6 & $\mathrm{M} / 55$ & Vasospastic angina pectoris & Chronic Af & Natural alpha & 480 & 6 months ${ }^{* *}$ \\
\hline 7 & $\mathrm{M} / 47$ & Vasospastic angina pectoris & None & Natural alpha & 480 & 11 months** \\
\hline 8 & $F / 54$ & Microvascular angina & Hypertension & Natural alpha & 138 & 23 days \\
\hline \multicolumn{7}{|c|}{ Myocardial disease } \\
\hline 9 & $F / 59$ & Cardiomyopathy & Hypertension & Natural alpha & 366 & 19 weeks* \\
\hline 10 & $F / 57$ & Cardiomyopathy & None & Recombinant alpha-2B & 270 & 45 days* \\
\hline
\end{tabular}

*Interferon therapy was discontinued; ${ }^{* *}$ cardiovascular adverse effect was observed after the end of treatment; IFN = interferon; $\mathrm{MU}=$ million units; $\mathrm{Af}=$ atrial fibrillation; $\mathrm{A}-\mathrm{V}$ block = atrioventricular block. 
Table II. History of Cardiovascular Diseases of the Study Population

\begin{tabular}{lrc}
\hline Hypertension & 55 & cases \\
Atrial premature contraction & 3 & 2 \\
Atrial fibrillation & 2 \\
Angina pectoris & 2 \\
Hypertrophic cardiomyopathy & 2 \\
Left ventricular dysfunction (mild) & 1 \\
Pacemaker for atrioventricular block & 1 \\
Ventricular premature contraction & 1 \\
Post operation for atrial septal defect & 1 \\
Post operation for ventricular septal defect & \\
\hline
\end{tabular}

provement of chest pain after taking vasodilators. The 2 patients with myocardial disease had cardiomyopathy; they showed transient left ventricular dysfunction. The clinical data of these 10 cases are presented in Table $I$.

Sex and age: There were 4 men and 6 women, with an average age of 56 years (range, 47 to 64). They were not significantly older than the rest of the study population.

History of cardiovascular disease: Fifty-five patients had hypertension and were administered antihypertensive drugs. Underlying cardiac diseases were recognized in 15 patients (Table II). Among the patients showing cardiovascular adverse effects of IFN, 4 had hypertension and one had chronic $A f$ before IFN therapy. This latter patient experienced angina pectoris 6 months after the end of IFN therapy (Case 6). The rate of cardiovascular disease before IFN therapy among these 10 cases was not different from that of those who did not develop cardiovascular adverse reactions.

Types and dose of IFN: Natural alpha IFN induced cardiovascular adverse reactions in 4 patients, recombinant alpha-2a in 4 and recombinant alpha-2b in 2. No adverse cardiovascular reactions were caused by beta IFN in this series, but the statistical significance of this finding was not recognized. The total dose of IFN varied greatly among the 6 patients who developed cardiovascular adverse reactions during IFN therapy; it ranged from 99 million units to 366 million units. The length of treatment also varied widely from 11 days to 19 weeks and no cardiovascular adverse effect was observed right after the initiation of therapy. Four episodes of cardiovascular adverse reactions were recognized within 1 year after the end of IFN therapy at about 4,6, 7 and 11 months from the end of IFN therapy. Among the 17 patients who underwent repeat IFN therapy, only 1 patient (Case 10) developed a cardiovascular adverse effect during the second course of therapy.

Countermeasures for cardiovascular adverse effects of IFN. IFN was discontinued immediately in five of the 6 patients who showed cardiovascular adverse reactions during IFN therapy. In the other patient, IFN therapy was contin- 


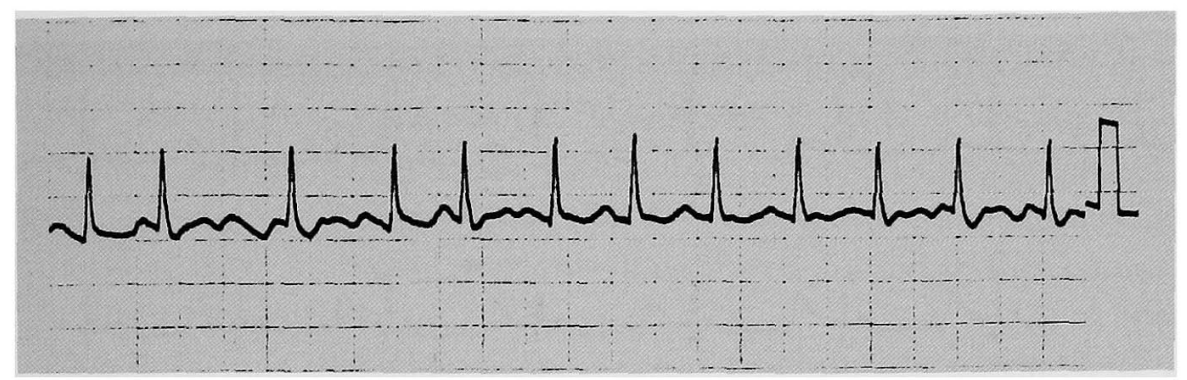

Figure 1. EGG (Case 1). This EGG taken when the patient felt palpitations, shows atrial fibrillation with rapid ventricular response.

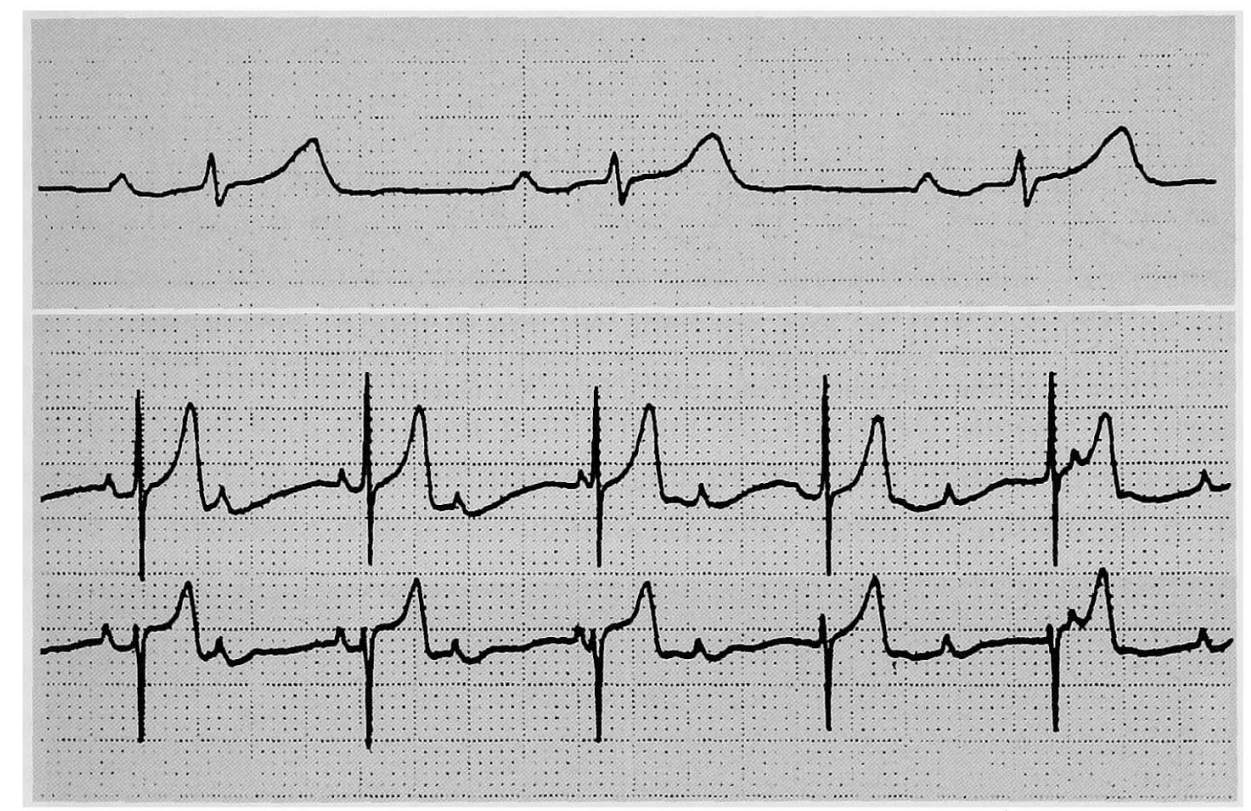

Figure 2. Holter EGG (Case 3). a) Holter ECG taken on October 1, 1993 showing first degree atrioventricular block. b) Holter ECG taken on November 1, the same year, showing complete atrioventricular block. ${ }^{8}$ )

ued together with antianginal agents (Case 8). In 4 of the 5 patients in whom IFN therapy was withdrawn, cardiovascular adverse reactions improved (Cases 1, 2, 4,9 ), while a temporary pacemaker and intraaortic balloon pumping were needed for the other patient (Case 10). Of the 4 patients who showed cardiovascular adverse reactions during follow up, one required a DDD-R pacemaker for complete A-V block; the other 3 received antianginal agents for angina pectoris and felt no chest symptoms thereafter.

Representative cases: Case 1. The patient, a 53-year-old woman, began treatment with 9 million units of recombinant alpha-2a IFN. On the 14th day of 


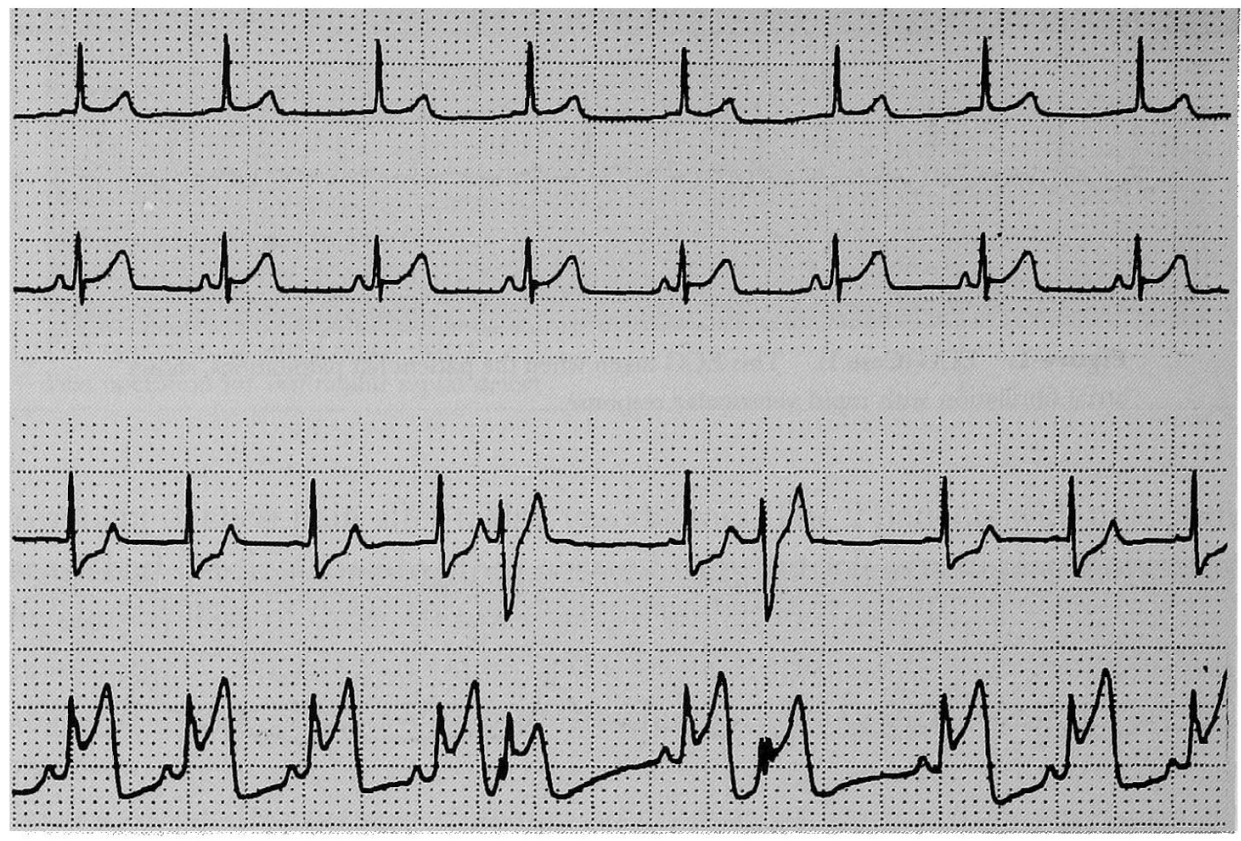

Figure 3. Holter ECG (Case 5). Holter ECG taken on March 25, 1993 showing no ST-T change when the patient had no pain (upper) and ST elevation at the same time the patient complained of chest pain at 04:40 AM (lower).
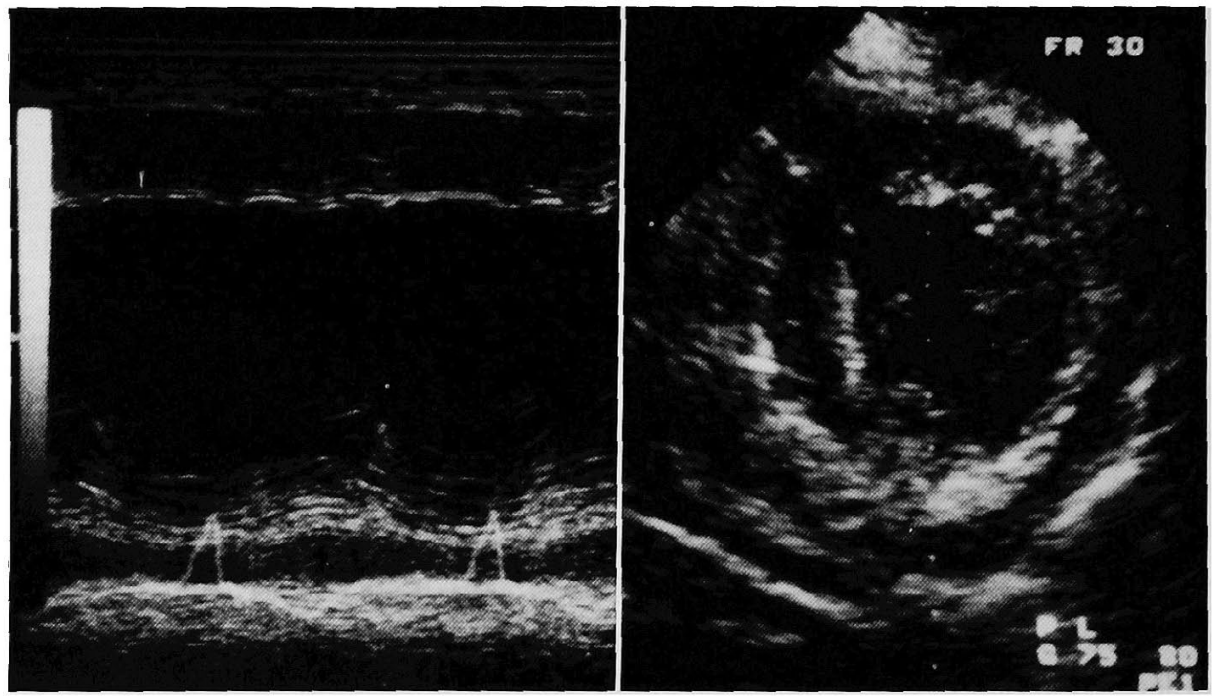

Figure 4. Echocardiogram (Case 10). The echocardiograms, short-axis parasternal view at the level of papillary muscles and M-mode at the same site showed thickening of the anterior wall of the left ventricle, reduced wall motion of the interventricular septum and anterior wall, and pericardial effusion in the shock. ${ }^{9}$ 
IFN therapy, she complained of palpitations and the ECG showed Af (Figure 1). The heart beat returned to its original rhythm after the patient was administered disopyramide $50 \mathrm{mg}$. Thereafter the patient experienced 4 more episodes of paroxysmal Af and depressive psychosis; thus IFN therapy was discontinued. After withdrawal of IFN, depressive psychosis disappeared and no further episodes of Af have been recognized.

Case 3. We have described this case before. ${ }^{8)}$ In brief, the patient, a 62-yearold woman, started treatment with recombinant alpha-2a IFN. She began to feel general fatigue on exertion during the treatment. The symptom continued after IFN therapy was completed. A Holter ECG revealed first degree A-V block (Figure 2-a). Her symptom worsened gradually and the Holter EGG taken 1 month later showed complete A-V block (Figure 2-b). A permanent pacemaker was implanted because her symptoms and complete $A-V$ block did not improve. After 1 year, abnormal shadows were recognized on the middle and lower fields of the right lung on the chest X-ray film and sarcoidosis was confirmed by thoracoscopic lung biopsy.

Case 5. The patient, a 62-year-old man, started treatment with recombinant alpha-2a IFN. About 7 months after the end of IFN therapy, he often experienced chest pain early in the morning. A Holter EGG revealed ST elevation associated with the chest pain (Figure 3). The CAG showed no organic stenosis. The diagnosis of vasospastic angina pectoris was established and he was started on treatment with a calcium-blocker, after which the patient felt no further chest symptoms.

Case 10 . We have described this case before. ${ }^{9)}$ In brief, this patient, a $57-$ year-old woman, started treatment with recombinant alpha-2b IFN. On the 46th day of therapy, her consciousness level declined suddenly. The ECG revealed complete $\mathrm{A}-\mathrm{V}$ block. The echocardiogram showed reduced motion of the interventricular septum and anterior wall, and pericardial effusion (Figure 4). Treatment for cardiogenic shock and the discontinuation of IFN therapy resulted in a remarkable improvement of cardiac function 3 weeks from the onset of symptoms. It is possible that the cardiogenic shock was due to acute myocarditis.

\section{Discussion}

In this series, arrhythmia, ischemic heart disease and myocardial disease were recognized as cardiovascular adverse effects of IFN during and after the end of treatment in patients with chronic hepatitis $\mathrm{C}$. These cardiovascular adverse effects, except for ventricular fibrillation, ${ }^{14,15,22)}$ myocardial infarction, ${ }^{15,20,21)}$ and sudden death, ${ }^{20)}$ which were not observed in our series, were very similar to those reported in previous studies in patients treated with IFN therapy for various 
malignancies. ${ }^{12-22)}$

Cardiovascular adverse effects were observed in $3.2 \%$ of patients in this study, while in patients with malignancies they were observed in $6 \%$ to $25 \%$ of the patients. ${ }^{15,19,20,22)}$ Cardiovascular adverse effects seemed to be seen less frequently in patients with chronic hepatitis $G$ than in those with malignancies. This difference might be due to the worse general condition of the patients with malignancies and to the combined antitumor drugs that might have affected the cardiovascular system in some cases.

Conceivable mechanisms: Various mechanisms that may explain the cardiovascular adverse effect of IFN therapy have been proposed. They include the inhibition of neogenesis of muscle-contracting protein, an autoimmune reaction, increased demand for oxygen, and coronary vasospasm..$^{12,23-25)}$ Moreover, it was recently shown that plasma levels of tumor necrosis factor, a cytokine, correlated with reduced contractility of the left ventricle ${ }^{26-28)}$ and impairment of the endothelium-dependent vascular rclaxation. ${ }^{29-31)}$ Therefore, exogenous IFN might cause myocardial diseasc or vasospastic angina pectoris through perturbation of the cytokine nctwork, including tumor necrosis factor ${ }^{32)}$ In addition, it is possible that not only one but multiple factors are involved in the development of a cardiovascular event and they might vary with the type of event. However, the exact pathogenic mechanism remains to be elucidated.

Time when the cardiovascular adverse reaction occurred: In this series, no cardiovascular adverse reaction occurred right after the initiation of IFN therapy. In the patients who experienced cardiovascular adverse effects during IFN therapy, these occurred from 11 days to 19 weeks after the start of treatment. Therefore, it seems appropriate to assume that cardiovascular adverse effects may occur at any time during IFN therapy.

We also recognized cardiovascular adverse effects $4,7,6$ and 11 months after the end of IFN therapy (Cases 3, 5 to 7). These were one case of A-V block and three of vasospastic angina pectoris. It is unclear whether these cardiovascular events were due to IFN itself. Regarding A-V block (Case 3) for instance, we considered that A-V block was not due to the direct influence of IFN, but was the first sign of sarcoidosis induced by IFN therapy. In this case, complete A-V block was recognized 4 months after the end of IFN therapy. Moreover, the adverse effects of IFN therapy, especially in autoimmune diseases, do not always improve after the cessation of IFN and sometimes persist for months, according to some reports. ${ }^{33,34)}$ Thus, some effects of IFN, including those mediated by the immune system, might sometimes continue for a few months after the end of treatment. This may explain why some of the patients developed cardiovascular adverse reactions a long time after stopping IFN therapy.

Prediction of cardiovascular adverse effects: All the patients who developed 
cardiovascular adverse reactions were treated with alpha type IFN; no cardiovascular adverse effects occurred in patients treated with beta IFN in this series. The reason why no cardiovascular adverse effects occurred in the patients treated with beta IFN remains unclear, but it might simply be because beta IFN was used in only a few patients.

Some reports have documented that patients with underlying heart diseases were prone to develop IFN-related cardiovascular complications, ${ }^{12,21)}$ but we found no relationship between cardiovascular adverse effects and the presence of previous cardiovascular disease in our series. Moreover, the relation between a cardiovascular adverse effect and clinical factors such as sex, age and the dose of IFN was evaluated, but no relation was established. Accordingly, the occurrence of cardiovascular adverse effects seemed to be unpredictable.

Countermeasures: Sonnenblick et a ${ }^{12)}$ reported that IFN therapy should be discontinued when patients develop severe or unstable heart disease; in case of mild, moderate or stable heart disease IFN therapy should be continued together with symptomatic treatment if necessary, while carefully monitoring the patients, and discontinued immediately when the disease becomes more severe. In fact, in most of the patients who experienced cardiovascular adverse effects during treatment in this series, IFN therapy was discontinued and the cardiovascular symptoms improved rapidly. However, considering that cardiovascular adverse effects also occurred after the end of IFN therapy, it seems that to discontinue IFN therapy alone is not enough and patients should be monitored for some time after withdrawal.

Study limitations: In this study, cardiovascular adverse effects were defined as a new finding recognized by various tests such as ECG, echocardiogram, treadmill exercise test and ambulatory electrocardiographic Holter recording in a patient who complained of no chest symptoms before IFN therapy. Moreover, only ECG and chest X-ray films were taken routinely before IFN therapy, and examination for underlying cardiovascular diseases was not exhaustive. This means that patients with asymptomatic cardiovascular adverse effects, such as arrhythmias, might have been undetected and the number of patients with potential cardiovascular diseases before IFN therapy, might be underestimated.

All cardiovascular diseases recognized during and after IFN therapy were described here. A-V block, which was due to sarcoidosis induced by IFN rather than to the direct effect of IFN, was also included. Among the IFN-related complications, the only one that may have any influence on the cardiovascular system was sarcoidosis and we included A-V block due to sarcoidosis in this series.

Conclusions: Adverse cardiovascular reactions such as arrhythmia, ischemic heart disease and myocardial disease occur frequently in patients with chronic 
hepatitis G treated with IFN. Cardiovascular adverse effects are unpredictable and they may occur even after the end of treatment. It is of great importance to monitor all patients for cardiovascular adverse effects not only during but also after the end of IFN therapy.

\section{REFERENCE}

1. Davis GL, Balart LA, Schiff ER, et al. Treatment of chronic hepatitis $\mathrm{C}$ with recombinant interferon alfa: A multicenter randomized, controlled trial. Hepatitis Interventional Therapy Group. N EnglJ Med 1989; 321: $1501-6$.

2. Hoofnagle JH, Di Bisceglie AM. Treatment of chronic type C hepatitis with alpha interferon. Semin Liver Dis 1989; 9: 259-63.

3. Renault PF, Hoofnagle JH. Side effects of alpha interferon. Semin Liver Dis 1989; 9: 273-7.

4. Saracco G, Touscoz A, Durazzo M, et al. Autoantibodies and response to alpha-interferon in patients with chronic viral hepatitis. J Hepatol 1990; 11: 339-43.

5. Kamisako $T$, Adachi $Y$, Chihara J, Yamamoto T. Interstitial pneumonitis and interferon-alfa. BMJ 1993; 306: 896.

6. Renault PF, Hoofnagle JH, Park Y, et al. Psychiatric complications of long-term interferon alfa therapy. Arch Intern Med 1987; 147: 1577-80.

7. Sartori M, Andorno S, La TG, et al. Assessment of interferon cardiotoxicity with quantitative radionuclide angiocardiography. Eur J Clin Invest 1995; 25: 68-70.

8. Teragawa $\mathrm{H}$, Hondo $\mathrm{T}$, Takahashi $\mathrm{K}$, et al. Sarcoidosis after interferon therapy for chronic active hepatitis C. Internal Medicine 1996; 35: 19-23.

9. Teragawa $\mathrm{H}, \mathrm{Hondo} \mathrm{T}$, Amano $\mathrm{H}$, et al. Cardiogenic shock following recombinant alpha-2b interferon therapy for chronic hepatitis C. Jpn Heart J 1996; 37: 137-42.

10. Kouno H, Aimitsu S, Ikemoto Y, Nakanishi T, Kajiyama G. Cardiovascular complications of interferon therapy in chronic hepatitis. C. Nippon Rinsho 1994; 52: 1914-8 (In Japanese with English abstract).

11. Tanaka H, Yamakado $T$, Emi $Y$, Nabeshima $K$, Itoh $S$, Nakano $T$. Interferon-induced coronary vasospasm: A case history. Angiology 1995; 46: 1139-43.

12. Sonnenblick M, Rosin A. Cardiotoxicity of interferon. A review of 44 cases. Chest 1991; 99: 557-61.

13. Kirkwood JM, Ernstoff MS. Interferons in the treatment of human cancer. J Clin Oncol 1984; 2: 33652.

14. Budd GT, Bukowski RM, Miketo L, Yen-Lieberman B, Proffitt MR. Phase-I trial of Ultrapure TM human leukocyte interferon in human malignancy. Cancer Chemother Pharmacol 1984; 12: 39-42.

15. Grunberg SM, Kempf RA, Itri LM, Vcnturi CL, Boswcll W Jr., Mitchell MS. Phase II study of recombinant alpha interferon in the treatment of advanced non-small cell lung carcinoma. Cancer Treat Rep 1985; 69: 1031-2.

16. Sonnenblick M, Rosenmann D, Rosin A. Reversible cardiomyopathy induced by interferon. BMJ 1990; 300: $1174-5$.

17. Schechter D, Nagler A. Recombinant interleukin-2 and recombinant interferon alpha immunotherapy cardiovascular toxicity. Am Heart J 1992; 123: 1736-9.

18. Gohen MG, Huberman MS, Nesto RW. Recombinant alpha 2 interferon-related cardiomyopathy. Am J Med 1988; 85: 549-51.

19. Merimsky O, Inbar M, Merimsky E, et al. Phase II study of recombinant interferon alpha-C in patients with metastatic renal cell carcinoma. Mol Biother 1990; 2: 155-9.

20. Cooper MR, Fefer A, Thompson J, et al. Alpha-2-interferon/melphalan/prednisone in previously untreated patients with multiple myeloma: a phase I-II trial. Cancer Treat Rep 1986; 70: 473-6.

21. Oldham RR. Toxic effects of interferon. Science 1982; 209: 902.

22. Ernstoff MS, Nair S, Bahnson RR, et al. A phase IA trial of sequential administration recombinant DNA-produced interferons: combination recombinant interferon gamma and recombinant interferon alfa in patients with metastatic renal cell carcinoma. J Clin Oncol 1990; 8: 1637-49. 
23. Blalock JE, Stanton JD. Common pathways of interferon and hormonal action. Nature 1980; 283: 4068.

24. Lampidis TJ, Brouty-Boye D. Interferon inhibits cardiac cell function in vitro. Proc Soc Exp Biol Med 1981; 166: 181-5.

25. Zbinden G. Effects of recombinant human alpha-interferon in a rodent cardiotoxicity model. Toxicol Lett 1990; 50: 25-35.

26. Balligand JL, Ungureanu D, Kelly RA, et al. Abnormal contractile function due to induction of nitric oxide synthesis in rat cardiac myocytes follows exposure to activated macrophage-conditioned medium. J Clin Inyest 1993; 91: 2314 9.

27. Yokoyama T, Vaca L, Rossen RD, Durante W, Hazarika P, Mann DL. Cellular basis for the negative inotropic effects of tumor necrosis factor- alpha in the adult mammalian heart. J Clin Invest 1993; 92 : 2303-12.

28. Finkel MS, Oddis CV, Jacob TD, Watkins SC, Hattler BG, Simmons RL. Negative inotropic effects of cytokines on the heart mediated by nitric oxide. Science 1992; 257:387-9.

29. Robert R, Chapelain B, Jean T, Neliat G. Interleukin-1 impairs both vascular contraction and relaxation in rabbit isolated aorta. Biochem Biophys Res Commun 1992; 182: 733-9.

30. Aoki N, Siegfried M, Lefer AM. Anti-EDRF effect of tumor necrosis factor in isolated, perfused cat carotid arteries. Am J Physiol 1989; 256: 1509-12.

31. Siegfried MR, Ma XL, Lefer AM. Splanchnic vascular endothelial dysfunction in rat endotoxemia: role of superoxide radicals. Eur J Pharmacol 1992; 212: 171-6.

32. Daniels HM, Meager A, Eddleston ALW, Alexander GM, Williams R. Spontaneous production of tumor necrosis factor $\alpha$ and interleukin-1 $\beta$ during interferon- $\alpha$ treatment of chronic $\mathrm{HBV}$ infection. Lancet $1990 ; 335: 875-7$.

33. Chin K, Tabata C, Sataka N, Nagai S, Moriyasu F, Kuno K. Pneumonitis associated with natural and recombinant interferon alfa therapy for chronic hepatitis C. Chest 1994; 105: 93941.

34. Watanabe N, Miura S, Yamaguchi E, Suzuki J, Kawakami Y. A case of interferon-alpha-induced pneumonitis. Nippon Kyobu Shikkan Gakkai Zasshi 1993; 31: 1308-12 (In Japanese with English abstract). 